\title{
Opposing Effects of the Anesthetic Propofol at Pentameric Ligand-Gated Ion Channels Mediated by a Common Site
}

\author{
Timothy Lynagh and Bodo Laube \\ Neurophysiology and Neurosensory Systems, Department of Biology, Technical University of Darmstadt, 64287 Darmstadt, Germany
}

Propofol is an intravenous general anesthetic that alters neuronal excitability by modulating agonist responses of pentameric ligandgated ion channels (pLGICs). Evidence suggests that propofol enhancement of anion-selective pLGICs is mediated by a binding site between adjacent subunits, whereas propofol inhibition of cation-selective pLGICs occurs via a binding site contained within helices M1-M4 of individual subunits. We considered this idea by testing propofol modulation of homomeric human glycine receptors (GlyRs) and nematode glutamate-gated chloride channels (GluCls) recombinantly expressed in Xenopus laevis oocytes with electrophysiology. The Haemonchus contortus AVR-14B GluCl was inhibited by propofol with an $\mathrm{IC}_{50}$ value of $252 \pm 48 \mu \mathrm{M}$, providing the first example of propofol inhibition of an anion-selective pLGIC. Remarkably, inhibition was converted to enhancement by a single I18'S substitution in the channel-forming M2 helix $\left(\mathrm{EC}_{50}=979 \pm 88 \mu \mathrm{M}\right)$. When a previously identified site between adjacent subunits was disrupted by the

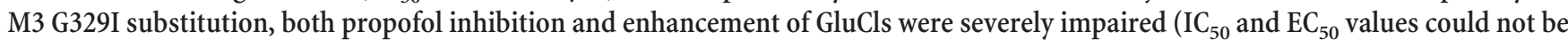
calculated). Similarly, when the equivalent positions were examined in GlyRs, the M2 S18'I substitution significantly altered the maximum level of enhancement by propofol, and the M3 A288I substitution abolished propofol enhancement. These data are not consistent with separate binding sites for the opposing effects of propofol. Instead, these data suggest that propofol enhancement and inhibition are mediated by binding to a single site in anion-selective pLGICs, and the modulatory effect on channel gating depends on the M2 $18^{\prime}$ residue.

\section{Introduction}

The general anesthetic propofol (2,6-diisopropylphenol) decreases neuronal excitability in humans by enhancing agonistinduced activation of type A GABA and glycine receptors $\left(\mathrm{GABA}_{\mathrm{A}} \mathrm{Rs}\right.$ and GlyRs), anion-selective members of the pentameric ligand-gated ion channel (pLGIC) family (Zeller et al., 2008; Nguyen et al., 2009). Photolabeling with propofol analogues (Yip et al., 2013), propofol inhibition of introduced cysteine labeling (Bali and Akabas, 2004), and conventional mutagenesis experiments (Krasowski et al., 1998) suggest that propofol binds between adjacent subunits in $\mathrm{GABA}_{\mathrm{A}} \mathrm{Rs}$ at a site overlapping that of the nonconventional agonist ivermectin in nematode glutamategated chloride channels (GluCls; Hibbs and Gouaux, 2011). In contrast, cation-selective 5-hydroxytryptamine-gated pLGICs (5-HT3Rs; Rüsch et al., 2007) and acetylcholine-gated pLGICs (nAChRs; Flood et al., 1997) are inhibited by propofol, and the only propofol/pLGIC crystal structure available shows propofol bound within membrane-spanning helices M1-M4 of individual subunits of GLIC, a bacterial cation-selective pLGIC that is inhibited by propofol (Nury et al., 2011). This raises the possibility

\footnotetext{
Received Oct. 8, 2013; revised Dec. 11, 2013; accepted Jan. 1, 2014.

Author contributions: T.L. designed research; T.L. performed research; T.L. and B.L. analyzed data; T.L. and B.L. wrote the paper.

The authors thank Michael Kilb and Stephan A. Pless for critical reading of the manuscript.

The authors declare no competing financial interests.

Correspondence should be addressed to Timothy Lynagh at the above address. E-mail: lynagh@bio.tu-darmstadt.de.

DOI:10.1523/JNEUROSCI.4307-13.2014

Copyright $\odot 2014$ the authors $\quad 0270-6474 / 14 / 342155-05 \$ 15.00 / 0$
}

that enhancement and inhibition of pLGICs are mediated by distinct propofol binding sites: enhancement by an intersubunit site; and inhibition by an intrasubunit site. In support of this idea, $\mathrm{x}$-ray crystallography and mutagenesis results at GLIC suggest that the intersubunit site by which enhancement occurs is naturally obscured by a phenylalanine side chain at the M2 14' position that is conserved in cation-selective pLGICs (Sauguet et al., 2013); GlyRs, by contrast, possess a smaller glutamine residue at this position, allowing propofol to bind between subunits and enhance channel gating (Sauguet et al., 2013).

These structural hypotheses were recently put to functional tests in GLIC. Propofol binding to GLIC increased accessibility to the site contained within single subunits (Ghosh et al., 2013), and mutations in this cavity did not impair propofol inhibition (Ghosh et al., 2013; Sauguet et al., 2013). Both of these results are inconsistent with a mechanism of inhibition in which propofol binds to a site within individual subunits. Thus, the question remains as to why propofol enhances or inhibits various pLGICs. In an attempt to address this question, we examined propofol modulation of two anion-selective pLGICs, the human $\alpha 1$ GlyR and the Haemonchus contortus AVR-14B GluCl. We discovered that the AVR-14B GluCl, unlike GlyRs and GABA ${ }_{A}$ Rs, is inhibited by propofol. Furthermore, a single M2 substitution that altered the efficacy of propofol enhancement of GlyRs converted propofol inhibition of AVR-14B GluCls to enhancement. This enabled us to test propofol-inhibited and propofol-enhanced AVR-14B GluCls for altered propofol sensitivity upon substitution of an M3 residue at the site between adjacent subunits. In GlyRs, propofol-inhibited GluCls, and propofol-enhanced 
GluCls, the M3 substitution dramatically impaired propofol modulation. These results point to a new explanation for the opposing effects of propofol modulation of pLGICs, in which propofol binds to a single site between subunits, and the effect on channel gating depends on a remote M2 position.

\section{Materials and Methods}

Reagents. Glycine, $\mathrm{NaCl}, \mathrm{KCl}, \mathrm{CaCl}_{2}, \mathrm{MgCl}_{2}$, HEPES, and dimethyl sulfoxide were purchased from Carl Roth; $\mathrm{NaOH}$ from AppliChem; and L-glutamate, propofol (2,6-diisopropylphenol), tricaine, gentamycin, and type IIA collagenase from Sigma-Aldrich. One mole stocks of glycine and L-glutamate were prepared in bath solution (see Oocyte preparation and electrophysiology). One mole stocks of propofol were prepared in dimethyl sulfoxide. Stocks were stored at $-20^{\circ} \mathrm{C}$, and dilutions were prepared directly before experiments. NotI and XbaI were purchased from New England Biolabs; the Quikchange II XL Site-Directed Mutagenesis kit from Agilent Technologies; and mMESSAGE mMACHINE transcription kits from Life Technologies.

Site-directed mutagenesis and cRNA synthesis. The human $\alpha 1$ GlyR subunit cDNA in the pNKS2 vector (Haeger et al., 2010) and the $H$. contortus AVR-14B (also called $\alpha 3 \mathrm{~B}$ and GBR-2B) GluCl subunit cDNA in the T7 vector (McCavera et al., 2009; kindly provided by Professor Adrian J. Wolstenholme, University of Georgia) were used for cRNA synthesis and site-directed mutagenesis. Mutant cDNAs were generated with the Quikchange II XL Site-Directed Mutagenesis kit. Sequences of entire GlyR or GluCl inserts were confirmed (Eurofins MWG Operon) and cDNAs were linearized with NotI (GlyR) or XbaI (GluCl) and transcribed with SP6 (GlyR) or T7 ( $\mathrm{GluCl}$ ) transcription kits.

Oocyte preparation and electrophysiology. Female Xenopus laevis frogs were anesthetized with $0.3 \%$ tricaine, and oocytes were surgically removed, in accordance with the Technical University of Darmstadt (Agreement V54-19c20/15 DA8/Anz. 20). Oocytes were transferred to ND96 solution with the following components (in mM): $96 \mathrm{NaCl}, 2 \mathrm{KCl}$, $1 \mathrm{CaCl}_{2}, 1 \mathrm{MgCl}_{2}, 5 \mathrm{HEPES}$, pH 7.4 with $\mathrm{NaOH}$, supplemented with 50 $\mathrm{mg} / \mathrm{ml}$ gentamycin. Stage V or VI oocytes were isolated and then defolliculated by $2 \mathrm{~h}$ incubation in type IIA collagenase. Oocytes were then rinsed with $\mathrm{Ca}^{2+}$-free ND96 for $10 \mathrm{~min}$ and stored in ND96 at $4^{\circ} \mathrm{C}$ until cRNA injection. Four nanograms of $100 \mathrm{ng} / \mu \mathrm{l} \mathrm{cRNAs}$ were injected into oocytes, which were then stored in ND96 at $18^{\circ} \mathrm{C}$ until experiments $2-4$ $\mathrm{d}$ later. In experiments, oocytes were continuously perfused in a plastic recording chamber with bath solution comprising the following components (in mM): $115 \mathrm{NaCl}, 1 \mathrm{KCl}, 1.8 \mathrm{CaCl}_{2}, 10$ HEPES, pH 7.4 with $\mathrm{NaOH}$. Oocytes were clamped at $-70 \mathrm{mV}$ by two-electrode voltage-clamp with microelectrodes containing $3 \mathrm{M} \mathrm{KCl}$. Currents were acquired at $200 \mathrm{~Hz}$ with a Geneclamp 500B amplifier, a Digidata 1322A digitizer, and Clampex 9.2 software (Molecular Devices). Glutamate or glycine, dissolved in bath solution, was applied alone or after $30 \mathrm{~s}$ preapplication of propofol, also dissolved in bath solution.

Data analysis. Currents were measured with Clampfit 9.2 software (Molecular Devices), and all subsequent data analyses, including statistical tests, were performed in Prism 4 (GraphPad Software). Peak current responses to glycine or glutamate were plotted against agonist concentration and fit with variable slope nonlinear regression to establish agonist $\mathrm{EC}_{50}$ values and Hill slope $\left(n_{\mathrm{H}}\right)$ parameters; mean \pm SEM are reported. For propofol enhancement, responses to $\mathrm{EC}_{20}$ glycine or $\mathrm{EC}_{20}$ glutamate after propofol application were divided by the response without propofol, and from this value, 1 was subtracted, giving "fold enhancement" reported in figures and text. In this way, a value of 0 equates to no effect of propofol on agonist responses. For propofol inhibition, responses to $\mathrm{EC}_{50}$ glutamate after propofol application were divided by the response without propofol, giving the remaining fractional current indicated in figures; in the main text, percentage inhibition of the control response is reported. Propofol-induced fold enhancement or remaining fractional current were fit with variable slope nonlinear regression (Prism 4), giving propofol $\mathrm{EC}_{50}$ or $\mathrm{IC}_{50}$ values and $n_{\mathrm{H}}$ parameters for each individual experiment; means \pm SEM for the different constructs are reported. In all experiments, each construct was tested in at least two batches of oocytes, alongside at least two other constructs. Means for mutants were compared with means for WT and means for glutamateinduced activation were compared with means for glutamate-induced activation in the presence of a single concentration of propofol by unpaired Student's $t$ test. Differences with a $p$ value $<0.05$ were considered significant; absolute $p$ values are reported in the main text.

Sequence alignment and homology modeling. All sequence alignments, including that in Figure 2 and those used for homology modeling were performed with ClustalW (Goujon et al., 2010). The H. contortus AVR14B GluCl (McCavera et al., 2009) model was based on the crystal structure of a truncated $\alpha \mathrm{GluCl}$ from Caenorhabditis elegans in complex with glutamate and ivermectin (PDB 3RIF; Hibbs and Gouaux, 2011). In generating this model, AVR-14B amino acids aligning with truncated segments of 3 RIF were removed from the AVR-14B sequence, leaving a truncated AVR-14B subunit of 338 aa with $61 \%$ sequence identity with 3RIF. The model was generated on the Swiss-Model homology modeling server (Arnold et al., 2006) and refined on the KoBaMIN server for knowledge-based energy minimization (Rodrigues et al., 2012). Refinement increased the Qualitative Model Energy Analysis (QMEAN) score for predicted reliability from 0.496 to 0.512 (Benkert et al., 2008). The refined structure was then assessed and figures were generated in PyMol v1.4.1 (Schrödinger). Figure 1 shows an $\alpha 1$ GlyR model that is also built on PDB 3RIF and was previously described (Lynagh et al., 2011).

\section{Results}

To address the possibility that size or polarity of the Q14' side chain determines propofol actions at anion-selective pLGICs, as raised by Sauguet et al. (2013), we tested the effects of propofol on glycine-gated currents at mutant Q14' F, Q14' $\mathrm{E}$, and Q14' $\mathrm{K} \alpha 1$ GlyRs (Fig. 1A). Propofol enhanced all mutant GlyRs (Fig. $1 A, B$ ), without differing significantly from the wild-type (WT) propofol $\mathrm{EC}_{50}$ value of $690 \pm 100 \mu \mathrm{M}, n_{\mathrm{H}}$ value of $2.4 \pm 0.4$, and maximal $3.0 \pm 0.2$-fold enhancement $(n=5$; all mutant values compared 
A

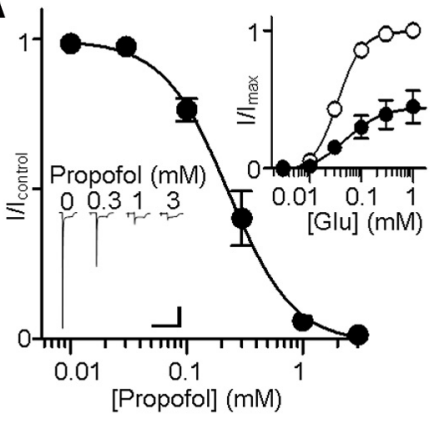

C

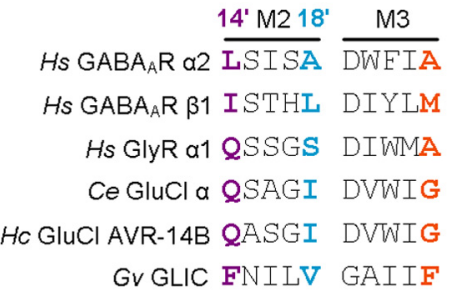

B

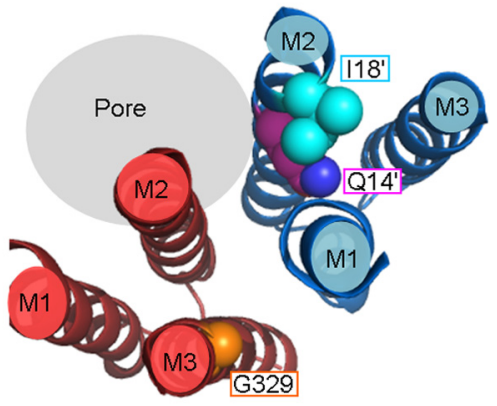

D Propofol $(\mathrm{mM})$

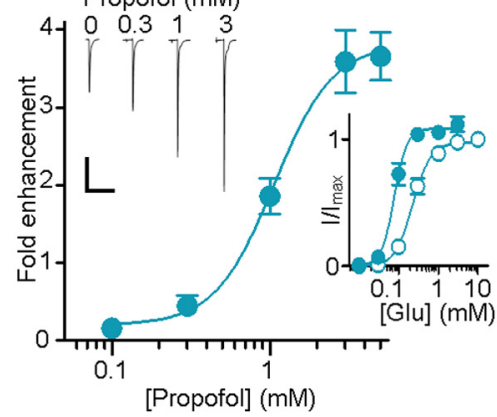

Figure 2. A single M2 mutation converts propofol inhibition of GluCls to enhancement. $A$, Glutamate-gated currents at WT GluCls are inhibited by propofol. A fixed glutamate concentration of $30 \mu \mathrm{m}$ was applied in the presence of propofol at increasing concentrations, as indicated. The glutamate dose-response relationship (right inset, hollow dot) is right-shifted and maximal currents are decreased in the presence of $300 \mu \mathrm{m}$ prop ofol (filled dot). All data points in this figure are mean \pm SEM. $B$, Membranespanning helices M1-M3 from two adjacent subunits in AVR-14B GluCl homology model, showing Q14', I18', and G329. C, Amino acid sequence alignment of Homo sapiens (Hs), C. elegans (Ce), H. contortus (Hc), and Gloeobacter violaceus (Gv) pLGIC subunits. Colors as in B. D, Glutamate-gated currents at I18'S GluCls are enhanced by propofol. A fixed glutamate concentration of 80-100 $\mu \mathrm{M}$ (according to the $\sim \mathrm{EC}_{20}$ glutamate concentration for the individual oocyte) was applied in the presence of propofol at increasing concentrations, as indicated. Right inset, Glutamate dose-response relationship without (hollow dots) and with 700 $\mu \mathrm{m}$ propofol (filled dots). Scale bars: $A, D, x, 1$ min; $y, 0.4 \mu \mathrm{A}$.

A

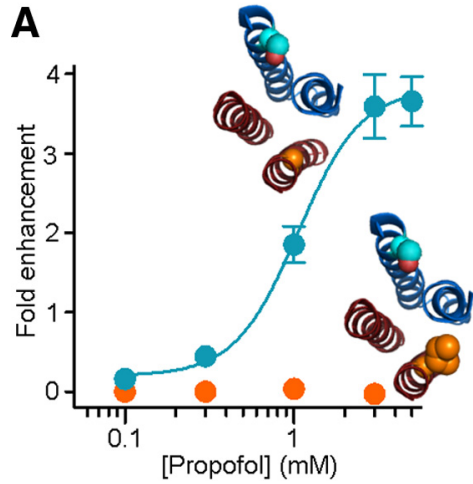

B

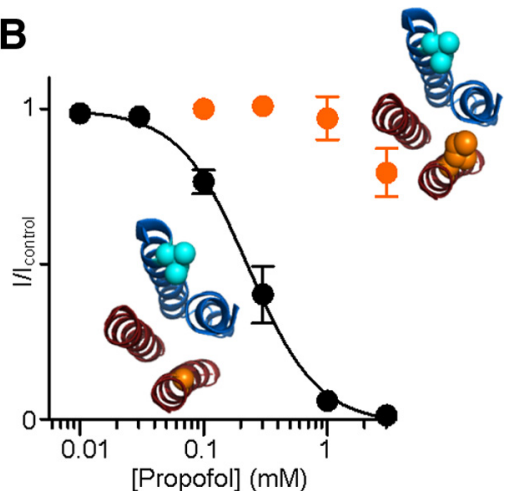

Figure 3. The M3 G329l substitution impairs both inhibition and enhancement of GluCls. $\boldsymbol{A}$, Propofol enhancement (mean \pm SEM) of mutant I18'S (blue dots) and lack of enhancement of mutant I18'S/G329I (orange dots) GluCls. B, Propofol inhibition of WT (black dots) and mutant G329I (orange dots) GluCls.

with WT value with Student's $t$ test), indicating that the $14^{\prime}$ side chain does not determine propofol enhancement of GlyRs. Given that M2 hydroxyl side chains pose a direct means for ligands to enhance channel gating of pLGICs (Hibbs and Gouaux, 2011), we considered other upper-M2 residues as possible determinants of propofol enhancement of GlyRs. We focused on the 18' position, as 18' mutations in GLIC alter propofol potency and channel gating (Nury et al., 2011; Ghosh et al., 2013) and the adjacent $17^{\prime}$ position binds ortho-propofol diazirine in $\mathrm{GABA}_{\mathrm{A}} \mathrm{Rs}$ (Yip et al., 2013). To test the requirement of a small polar side chain at this position for propofol enhancement, we generated mutant S18'I GlyRs. These were enhanced with significantly greater potency than WT GlyRs, with an $\mathrm{EC}_{50}$ value of $132 \pm 29$ $\mu \mathrm{M}(n=5 ; p=0.006)$ and maximal $4.7 \pm$ 0.7 -fold enhancement ( $p=0.029$; Fig. $1 A, B ; n_{\mathrm{H}}=2.6 \pm 0.3$, not significantly different from $W T$ ). This indicates that although a small polar side chain at the $18^{\prime}$ position is not required for propofol enhancement, this position may be allosterically involved in coupling propofol binding to channel gating. An allosteric involvement of $S 18^{\prime}$ in propofol actions is supported by the distance separating S18' from a putative propofol binding cavity between subunits that includes the M3 A288 residue (Fig. 1C). To verify these ideas, we tested mutant A288I and S18'I/ A288I GlyRs for propofol enhancement of glycine-gated currents. At both of these mutants, glycine-gated currents were insensitive to propofol at concentrations up to $10 \mathrm{~mm}(n=4$; Fig. $1 A, B)$, which is consistent with a direct role of A288 in propofol binding and an allosteric role of S18 in propofol enhancement of channel gating.

We next considered possible mechanisms of propofol modulation of invertebrate GluCls, given the structural template of the Caenorhabditis elegans $\alpha \mathrm{GluCl}$ crystal structure (Hibbs and Gouaux, 2011) and the accuracy with which $\mathrm{GluCl}$ modulation can describe human pLGIC modulation (Hibbs and Gouaux, 2011; Lynagh et al., 2011). We therefore tested propofol modulation of the H. contortus AVR-14B $\mathrm{GluCl}$, which is $66 \%$ identical to the $C$. elegans $\alpha \mathrm{GluCl}$ regarding M1-M4 helices and possesses the advantage of robust responses to the agonist glutamate (Cully et al., 1994; McCavera et al., 2009). In a similar concentration range to its enhancement of GlyRs, propofol inhibited glutamate-gated currents at GluCls (Fig. $2 A$ ), with an $\mathrm{IC}_{50}$ value of $252 \pm 48 \mu \mathrm{M}$ and $n_{\mathrm{H}}$ value of $2.4 \pm 0.5(n=6)$. The presence of $300 \mu \mathrm{M}$ propofol shifted the glutamate $\mathrm{EC}_{50}$ value from $30 \pm 2 \mu \mathrm{M}$ to $60 \pm 12 \mu \mathrm{M}(n=4 ; p=0.037)$, decreased the maximal glutamate-induced current to $43 \pm 4 \%$ initial levels ( $p=0.040$; Fig. $2 A$ ), and did not significantly affect the glutamate $n_{\mathrm{H}}$ value (1.6 \pm 0.3 without propofol; $1.3 \pm 0.1$ with propofol). These data provide the first example of propofol inhibition of an anion-selective pLGIC. GluCls possess a Q14' residue like GlyRs (Fig. 2B,C), in line with the idea that the $14^{\prime}$ side chain does not determine propofol modulation of anion-selective pLGICs. The $18^{\prime}$ isoleucine, however, contrasts the smaller serine or alanine residue at this position in GlyR and $\mathrm{GABA}_{\mathrm{A}} \mathrm{R} \alpha$ subunits and more closely reflects the $18^{\prime}$ valine in 
GLIC (Fig. $2 B, C$ ). To test whether the $18^{\prime}$ side chain allosterically mediates propofol modulation of GluCls, as was the case in GlyRs, we tested the effects of propofol on glutamate-gated currents at mutant I18'S AVR-14B GluCls. Remarkably, inhibition was converted to enhancement (Fig. $2 D$ ), with an $\mathrm{EC}_{50}$ value of $979 \pm 88 \mu \mathrm{M}, n_{\mathrm{H}}$ value of $2.1 \pm 0.3$, and maximal 3.6 \pm 0.4 -fold enhancement $(n=7)$. Seven hundred micromole propofol shifted the glutamate $\mathrm{EC}_{50}$ value of $108 \pm 13 \mu \mathrm{M}$ to $41 \pm 5 \mu \mathrm{M}$ at I18'S GluCls $(n=4 ; p=0.003$; Fig. $2 D)$ without increasing maximal glutamate-gated currents or significantly affecting the $n_{\mathrm{H}}$ value of $1.8 \pm 0.2$, indicating that propofol enhances only submaximal current responses or increases apparent agonist affinity in I18'S GluCls. This reflects the leftward shift propofol induces in glycine and GABA dose-response relationships in human pLGICs (Orser et al., 1994; O'Shea et al., 2004).

To test whether propofol enhancement of mutant I18'S GluCls occurs via a similar mechanism as in GlyRs and $\mathrm{GABA}_{\mathrm{A}} \mathrm{Rs}$, we tested the effects of the G329I substitution, as mutations at the equivalent position in GlyRs (A288; present study) and $\mathrm{GABA}_{\mathrm{A}}$ Rs (Krasowski et al., 2001) abolish propofol enhancement. We generated I18'S/G329I GluCls and found that at concentrations $\leq 3 \mathrm{~mm}$, propofol had no effect on glutamate-gated currents (Fig. 3A). This result is consistent with propofol enhancement occurring by propofol binding to a site between adjacent subunits. In a final test, we sought to compare propofol inhibition of WT GluCls and propofol enhancement of I18'S GluCls in terms of their structural requirements at the putative binding site between subunits. We reasoned that if binding sites for enhancement and inhibition were different, the G329I substitution alone would have no effect on propofol inhibition of GluCls. However, G329I GluCls showed dramatically decreased propofol sensitivity, with $3 \mathrm{~mm}$ propofol inhibiting only $12 \pm 7 \%$ of the $\mathrm{EC}_{50}$ glutamate-induced current (Fig. $3 B$ ), suggesting that, like propofol enhancement, propofol inhibition depends on a site between adjacent subunits.

\section{Discussion}

In the present study, we found that in two different anionselective pLGICs, substitution of the M2 18' residue altered the efficacy with which propofol modulates agonist responses, to the extent that inhibition was converted to enhancement in the AVR14B GluCl. Also, substitution of an M3 alanine (in the $\alpha 1$ GlyR) or M3 glycine (in the AVR-14B GluCl) at the intersubunit site abolished enhancement and severely impaired inhibition of WT and/or mutant receptors. The simplest interpretation of the present results is that propofol binds to a single intersubunit site in anionic pLGICs, and the efficacy of modulation depends on the M2 18' residue. This interpretation relies heavily on the assumption that impaired propofol sensitivity upon M3 mutations is a result of an altered binding site and that the altered efficacy of propofol modulation upon M2 mutations is due to altered channel gating, but it is consistent with previous experiments at $\mathrm{GABA}_{\mathrm{A}} \mathrm{Rs}$ and is supported by the actions of ivermectin at anionselective pLGICs. In $\mathrm{GABA}_{\mathrm{A}} \mathrm{Rs}$, propofol inhibits the labeling of an introduced cysteine at the equivalent M3 position (Bali and Akabas, 2004) and the binding of azietomidate to M3 and M1 residues at the intersubunit site (Li et al., 2010). Ivermectin binds between helices of adjacent subunits in GluCls (Hibbs and Gouaux, 2011) and GlyRs (Lynagh et al., 2011), and whereas $\alpha 1$ GlyR A288 and AVR-14B GluCl G329 mutations abolish ivermectin enhancement and/or activation (Lynagh and Lynch, 2010; Lynagh et al., 2011), $\alpha 1$ GlyR M2 side chain substitutions can alter the direction of modulation without affecting the concentrations required for modulation (Lynagh et al., 2011).

New knowledge of propofol inhibition of GluCls and of the allosteric role of the $18^{\prime}$ residue in propofol modulation sheds some light on the possible roles of other residues. Figure $2 \mathrm{C}$ shows that both $\alpha 1$ GlyRs, which are enhanced by propofol, and AVR-14B GluCls, which are inhibited, both possess serine and glycine residues at the M2 $16^{\prime}$ and $17^{\prime}$ residues, respectively. These two positions are therefore unlikely to determine whether propofol binding enhances or inhibits agonist-gated currents. The $18^{\prime}$ residue thus plays a specific role in linking propofol binding to agonist-gated currents, perhaps reflected in the increased agonist $\mathrm{EC}_{50}$ values at $\mathrm{GlyR}$ and $\mathrm{GluCl} 18^{\prime}$ mutants. A critical role for the upper end of M2 is also consistent with the labeling of M2 17' in $\mathrm{GABA}_{\mathrm{A}}$ Rs by ortho-propofol diazirine (Yip et al., 2013) and the negligible effects of M2 15' mutations on $\alpha 1$ GlyR modulation by propofol (Ahrens et al., 2008). Although the intersubunit site is significantly larger than a single propofol molecule (Murail et al., 2011), the present results suggest that lower residues of this site can be excluded as the principal determinants of propofol binding and modulation, which is consistent with the suggestions of Yip et al. (2013) regarding $\mathrm{GABA}_{\mathrm{A}} \mathrm{Rs}$.

Propofol enhancement or inhibition of different anionselective pLGICs via a single site in the membrane-spanning domain opens the door to bidirectional pharmacological targeting of different pLGIC subtypes via a conserved binding site. This may even apply to cation-selective nAChRs and $5-\mathrm{HT}_{3} \mathrm{Rs}$, given the recent findings that propofol inhibition of GLIC is unlikely to occur via the intrasubunit binding site (Ghosh et al., 2013) and that nAChRs possess both intrasubunit and intersubunit binding sites for propofol analogues (Jayakar et al., 2013).

\section{References}

Ahrens J, Leuwer M, Stachura S, Krampfl K, Belelli D, Lambert JJ, Haeseler G (2008) A transmembrane residue influences the interaction of propofol with the strychnine-sensitive glycine alphal and alphalbeta receptor. Anesth Analg 107:1875-1883. CrossRef Medline

Arnold K, Bordoli L, Kopp J, Schwede T (2006) The SWISS-MODEL workspace: a web-based environment for protein structure homology modelling. Bioinformatics 22:195-201. CrossRef Medline

Bali M, Akabas MH (2004) Defining the propofol binding site location on the GABAA receptor. Mol Pharmacol 65:68-76. CrossRef Medline

Benkert P, Tosatto SC, Schomburg D (2008) QMEAN: a comprehensive scoring function for model quality assessment. Proteins 71:261-277. CrossRef Medline

Cully DF, Vassilatis DK, Liu KK, Paress PS, Van der Ploeg LH, Schaeffer JM, Arena JP (1994) Cloning of an avermectin-sensitive glutamate-gated chloride channel from Caenorhabditis elegans. Nature 371:707-711. CrossRef Medline

Flood P, Ramirez-Latorre J, Role L (1997) Alpha 4 beta 2 neuronal nicotinic acetylcholine receptors in the central nervous system are inhibited by isoflurane and propofol, but alpha 7-type nicotinic acetylcholine receptors are unaffected. Anesthesiology 86:859-865. CrossRef Medline

Ghosh B, Satyshur KA, Czajkowski C (2013) Propofol binding to the resting state of the gloeobacter violaceus ligand-gated ion channel (GLIC) induces structural changes in the inter- and intrasubunit transmembrane domain (TMD) cavities. J Biol Chem 288:17420-17431. CrossRef Medline

Goujon M, McWilliam H, Li W, Valentin F, Squizzato S, Paern J, Lopez R (2010) A new bioinformatics analysis tools framework at EMBL-EBI. Nucleic Acids Res 38:W695-699. CrossRef Medline

Haeger S, Kuzmin D, Detro-Dassen S, Lang N, Kilb M, Tsetlin V, Betz H, Laube B, Schmalzing G (2010) An intramembrane aromatic network determines pentameric assembly of Cys-loop receptors. Nat Struct Mol Biol 17:90-98. CrossRef Medline

Hibbs RE, Gouaux E (2011) Principles of activation and permeation in an anion-selective Cys-loop receptor. Nature 474:54-60. CrossRef Medline 
Jayakar SS, Dailey WP, Eckenhoff RG, Cohen JB (2013) Identification of propofol binding sites in a nicotinic acetylcholine receptor with a photoreactive propofol analog. J Biol Chem 288:6178-6189. CrossRef Medline

Krasowski MD, Koltchine VV, Rick CE, Ye Q, Finn SE, Harrison NL (1998) Propofol and other intravenous anesthetics have sites of action on the gamma-aminobutyric acid type A receptor distinct from that for isoflurane. Mol Pharmacol 53:530-538. Medline

Krasowski MD, Nishikawa K, Nikolaeva N, Lin A, Harrison NL (2001) Methionine 286 in transmembrane domain 3 of the GABAA receptor beta subunit controls a binding cavity for propofol and other alkylphenol general anesthetics. Neuropharmacology 41:952-964. CrossRef Medline

Li GD, Chiara DC, Cohen JB, Olsen RW (2010) Numerous classes of general anesthetics inhibit etomidate binding to gamma-aminobutyric acid type A (GABAA) receptors. J Biol Chem 285:8615-8620. CrossRef Medline

Lynagh T, Lynch JW (2010) A glycine residue essential for high ivermectin sensitivity in Cys-loop ion channel receptors. Int J Parasitol 40:14771481. CrossRef Medline

Lynagh T, Webb TI, Dixon CL, Cromer BA, Lynch JW (2011) Molecular determinants of ivermectin sensitivity at the glycine receptor chloride channel. J Biol Chem 286:43913-43924. CrossRef Medline

McCavera S, Rogers AT, Yates DM, Woods DJ, Wolstenholme AJ (2009) An ivermectin-sensitive glutamate-gated chloride channel from the parasitic nematode Haemonchus contortus. Mol Pharmacol 75:1347-1355. CrossRef Medline

Murail S, Wallner B, Trudell JR, Bertaccini E, Lindahl E (2011) Microsecond simulations indicate that ethanol binds between subunits and could stabilize an open-state model of a glycine receptor. Biophys J 100:16421650. CrossRef Medline

Nguyen HT, Li KY, daGraca RL, Delphin E, Xiong M, Ye JH (2009) Behav- ior and cellular evidence for propofol-induced hypnosis involving brain glycine receptors. Anesthesiology 110:326-332. CrossRef Medline

Nury H, Van Renterghem C, Weng Y, Tran A, Baaden M, Dufresne V, Changeux JP, Sonner JM, Delarue M, Corringer PJ (2011) X-ray structures of general anaesthetics bound to a pentameric ligand-gated ion channel. Nature 469:428-431. CrossRef Medline

Orser BA, Wang LY, Pennefather PS, MacDonald JF (1994) Propofol modulates activation and desensitization of $\mathrm{GABA}_{\mathrm{A}}$ receptors in cultured murine hippocampal neurons. J Neurosci 14:7747-7760. Medline

O'Shea SM, Becker L, Weiher H, Betz H, Laube B (2004) Propofol restores the function of "hyperekplexic" mutant glycine receptors in Xenopus oocytes and mice. J Neurosci 24:2322-2327. CrossRef Medline

Rodrigues JP, Levitt M, Chopra G (2012) KoBaMIN: a knowledge-based minimization web server for protein structure refinement. Nucleic Acids Res 40:W323-W328. CrossRef Medline

Rüsch D, Braun HA, Wulf H, Schuster A, Raines DE (2007) Inhibition of human $5-\mathrm{HT}(3 \mathrm{~A})$ and $5-\mathrm{HT}(3 \mathrm{AB})$ receptors by etomidate, propofol and pentobarbital. Eur J Pharmacol 573:60-64. CrossRef Medline

Sauguet L, Howard RJ, Malherbe L, Lee US, Corringer PJ, Adron Harris RA, Delarue M (2013) Structural basis for potentiation by alcohols and anaesthetics in a ligand-gated ion channel. Nat Commun 4:1697. CrossRef Medline

Yip GM, Chen ZW, Edge CJ, Smith EH, Dickinson R, Hohenester E, Townsend RR, Fuchs K, Sieghart W, Evers AS, Franks NP (2013) A propofol binding site on mammalian GABA receptors identified by photolabeling. Nat Chem Biol 9:715-720. CrossRef Medline

Zeller A, Jurd R, Lambert S, Arras M, Drexler B, Grashoff C, Antkowiak B, Rudolph U (2008) Inhibitory ligand-gated ion channels as substrates for general anesthetic actions. In: Modern anesthetics (Schüttler J, Schwilden $\mathrm{H}$, eds), pp 31-51. Berlin: Springer. 